LBNL-43734

hep-th/9907101

\title{
Supersymmetric Quantum Mechanical Description of Four Dimensional Black Holes
}

\author{
Julie D. Blum \\ Theoretical Physics Group, Mail Stop 50A-5101 \\ Ernest Orlando Lawrence Berkeley National Laboratory \\ Berkeley, CA 94720 USA
}

\begin{abstract}
By assuming the existence of a novel multipronged string state for D-particles interacting with D-brane intersections in type IIA string theory, we are able to derive a quantum mechanical description of supersymmetric Reissner-Nordstrom black holes. A supersymmetric index calculation provides evidence for this conjecture. The quantum mechanical system becomes two decoupled conformal quantum mechanical systems in the low energy limit. The conformal quantum mechanics has expected properties of a dual description of string theory on $A d S_{2} \times S^{2}$.
\end{abstract}

$6 / 99$ 


\section{Introduction}

Following work describing the near horizon geometry of certain string $(\mathrm{M})$ theory black holes composed of solitonic branes as the maximally supersymmetric product of Anti de Sitter space and a sphere $(A d S \times S)$ with a conformal theory on the boundary [1], work that identified certain of the solitonic black holes as Dirichlet(D)-branes [2], work determining the low energy theory of D-branes to be a nonabelian gauge theory [3] known to be conformal in certain cases, and work showing that calculations of the properties of D-brane black holes could be performed successfully in the conformal theory for an appropriately large number of D-branes [4][5]; there was a conjecture [6] that supergravity or string (M) theory in the near horizon $A d S$ geometry of the solitonic (D-) branes was equivalent to the conformal theory on these branes. Further work gave a recipe for comparing the two theories and provided some evidence for the conjecture's validity in the supergravity limit [7] [8]. Whether or not all of the interesting aspects of string theory can be reduced to a field theory, one can at least derive some useful relations between the two theories following the above works.

The aim of this paper is to extend the relation to four-dimensional black holes with a near horizon geometry of $A d S_{2} \times S^{2}$. We will show that the two-dimensional conformal theory descriptions of the onebrane-fivebrane black hole [4] and generalizations [9] [10] [11] are alternatively described at low energies by a quantum mechanics that becomes conformal in the very low energy limit. Evidence will be presented that this quantum mechanics contains the degrees of freedom responsible for the ground state entropy of the black holes. The quantum mechanics will not describe completely the moduli space of the transverse six-fold, for we will assume that the local geometry of the D-particle is flat, and we will also neglect background fields. A two-dimensional description [12] [4] [11] may be better suited for this purpose although one could further complicate the quantum mechanics. On the other hand, to understand macroscopic features of the four-dimensional black hole, this quantum mechanics may be a reasonable approach. In the course of obtaining the quantum mechanical theory, we will propose some novel string states occurring at the intersections of D-branes. We hope that this proposal leads to a better understanding of these intersections. The rules we will develop are somewhat ad hoc but seem to lead to a sensible description.

The outline of the rest of this paper is as follows. In section two we will review some macroscopic properties as well as the microscopic effective string formulation of the 
black holes to be discussed. In section three we will present the novel string states that we believe to capture the low energy degrees of freedom of the black holes and a prescription for obtaining these states from the intersections of D-branes. In section four we will calculate the index of supersymmetric ground states [13] of the quantum mechanics in the simplest theory containing these states. We will extrapolate from this result a conjecture for the degeneracy of the large number of intersections case. The resulting ground state entropy will agree with the macroscopic and string formulation predictions. In section five we will derive the quantum mechanical system describing the black holes. We will take the low energy limit and obtain a conformal quantum mechanics. What is interesting here is that in this limit we appear to have two decoupled conformal quantum mechanical systems, a "Coulomb" branch with manifest $S O(3)$ symmetry and a "Higgs" branch with a large internal symmetry. However, these two branches are coupled in the full nonconformal theory. In section six we present our conclusions and directions for further research.

\section{Black Holes and Effective Strings}

\subsection{Review of Macroscopic Black Holes}

The four dimensional black holes we will consider in this paper are all extremal and of the Reissner-Nordstrom type. The metric takes the following form:

$$
d s^{2}=-\left(T_{1} T_{2} T_{3} T_{4}\right)^{1 / 2} d t^{2}+\left(T_{1} T_{2} T_{3} T_{4}\right)^{-1 / 2}\left(d r^{2}+r^{2} d \Omega_{2}^{2}\right)
$$

where $T_{i}=\left(1+Q_{i} / r\right)^{-1}$ and the $Q_{i}$ are positive. There are electric and magnetic fields,

$$
F_{i}=d t \wedge d T_{i}+* d T_{i}^{-1}
$$

The mass of the black hole is

$$
M=\frac{\sum_{i} Q_{i}}{4 G_{N}}
$$

with $G_{N}$ the four-dimensional Newton constant. For equal charges $Q$ the Ricci scalar vanishes and

$$
R_{\mu \nu} R^{\mu \nu}=\frac{4 Q^{4}}{(r+Q)^{4}}
$$

so there is no singularity in the extremal limit. The extremal entropy is

$$
S=\frac{\pi\left(Q_{1} Q_{2} Q_{3} Q_{4}\right)^{1 / 2}}{G_{N}}
$$


In the near horizon Reissner-Nordstrom reduces to $A d S_{2} \times S^{2}$ with metric

$$
d s_{N H}^{2}=\frac{-r^{2}}{\left(Q_{1} Q_{2} Q_{3} Q_{4}\right)^{1 / 2}} d t^{2}+\frac{\left(Q_{1} Q_{2} Q_{3} Q_{4}\right)^{1 / 2}}{r^{2}} d r^{2}+\left(Q_{1} Q_{2} Q_{3} Q_{4}\right)^{1 / 2} d \Omega_{2}^{2}
$$

while the metric at infinity is flat. The Ricci scalar of $A d S_{2}$ is $R=\frac{-2}{\left(Q_{1} Q_{2} Q_{3} Q_{4}\right)^{1 / 2}}$, and the cosmological constant is $\Lambda=\frac{1}{2} R$ while $R_{S^{2}}=2 \Lambda_{S^{2}}=-R_{A d S_{2}}$. There are numerous papers that have studied the Reissner-Nordstrom metric as a solution of string (M) theory. In type IIB string theory in a purely threebrane background, the equation to solve is

$$
R_{\mu \nu}=F_{\mu a_{1} a_{2} a_{3} a_{4}} F_{\nu}^{a_{1} a_{2} a_{3} a_{4}}
$$

where we have distinguished four-dimensional and six-fold indices in an obvious way, and $F$ is the five-form field strength. In the simplest case (a six-torus), one can reverse the signs of some components of the field strength while retaining a solution of the low energy field theory. Some of these reversals will break supersymmetry, and it is interesting to consider these black holes. We will comment on the consequences of breaking supersymmetry in this way in the next section.

\subsection{D-branes and Microscopic Strings}

The paradigmatic extremal onebrane-fivebrane black hole [4] is five-dimensional with near horizon geometry $A d S_{2} \times S^{3}$. Upon compactification on a circle the geometry is again Reissner-Nordstrom. This black hole has three charges. In the D-brane approach, these charges are the number $N_{5}$ of fivebranes wrapped on $K 3 \times S^{1}$, the number $N_{1}$ of onebranes wrapped on $S^{1}$, and the momentum $p=N_{0} / R$ with $R$ the radius of the $S^{1}$ and $N_{0}$ an integer. At low energies there is an effective conformal theory on $S^{1} \times$ time with central charge $c=6 N_{1} N_{5}$. The momentum $N_{0}$ corresponds to the eigenvalue of the Virasoro generator $L_{0}$. The sign of $N_{0}$ is not crucial here as the theory is left-right symmetric, but the signs of $N_{1}$ and $N_{5}$ are correlated. The entropy has been calculated [4] in the limit of large charges using the metric of the supergravity solution and alternatively the asymptotic microscopic formula for the degeneracy [14],

$$
d\left(N_{0}, c\right)=\exp 2 \pi \sqrt{\frac{N_{0} c}{6}}
$$

where $d$ is the degeneracy, and the entropy $S=\ln d=2 \pi \sqrt{\frac{N_{0} c}{6}}$. The two calculations of the entropy agree. The $K 3$ can be replaced by a $T^{4}$ [15] 16] with similar results. For the $T^{4}$ case one can choose any combination of signs for the three charges. 
By a sequence of U-duality operations we can convert the $T^{4}$ case to an $\mathrm{M}$ theory configuration. Compactify on a circle to four dimensions. Perform a T-duality on three directions-the newly compactified direction and two directions of $T^{4}$ (avoiding the momentum circle). Interchange the $\mathrm{M}$ theory circle and the momentum circle. The result is $N_{1} N_{5}$ fourbrane intersections on a two-torus and $N_{0}$ zerobranes. We presumably can play the same game with $K 3$ using mirror symmetry but the analysis seems more complicated for this case. In the latter part of this paper we will derive an effective quantum mechanics for the D-particles at the intersections.

The other prototypical Reissner-Nordstrom black hole solution of string theory has been discussed by [9] [10] and many others. In type IIB string theory the four charges $N_{i}$ are due to threebranes wrapped on a $T^{6}$ so that any two sets $(i, j)$ intersect in a string while three or four sets intersect in a point. There are therefore six strings along each direction $\left(T_{(i)}^{3} \cap T_{(j)}^{3}\right)$ of the six-torus and a total of $N_{1} N_{2} N_{3} N_{4}$ intersections on $T_{(1)}^{3} \cap T_{(2)}^{3} \cap T_{(3)}^{3} \cap T_{(4)}^{3}$. The supersymmetries which are preserved satisfy the following conditions:

$$
\begin{aligned}
& \Gamma_{11} \epsilon_{L}=\epsilon_{L} \\
& \Gamma_{11} \epsilon_{R}=\epsilon_{R} \\
& \Gamma_{0 a b c}^{i} \epsilon= \pm i \epsilon
\end{aligned}
$$

where $\Gamma_{11}=\Gamma_{0} \Gamma_{1} \ldots \Gamma_{10}$ with $\Gamma_{a}$ a ten-dimensional Clifford algebra matrix, $\Gamma_{0 a b c}^{i}=$ $\Gamma_{0} \Gamma_{a} \Gamma_{b} \Gamma_{c}$ where $a, b, c$ are the directions on $T^{6}$ on which the $N_{i}$ branes are wrapped, $\epsilon_{L}$ and $\epsilon_{R}$ are the two supersymmetries of type IIB from left and right movers of the string, and $\epsilon=\epsilon_{L}+i \epsilon_{R}$. The sign of the last relation depends on the sign of $N_{i}$. The $\Gamma^{i}$ commute and satisfy $\Gamma^{1} \Gamma^{2} \Gamma^{3} \Gamma^{4}= \pm 1$, and the number of preserved supersymmetries is

$$
N=\operatorname{tr}\left[\frac{1 \pm i \Gamma^{1}}{2} \frac{1 \pm i \Gamma^{2}}{2} \frac{1 \pm i \Gamma^{3}}{2} \frac{1 \pm i \Gamma^{4}}{2}\right] .
$$

Thus, $N=4$ or $N=0$. Regardless of the signs, any triple intersection preserves supersymmetry, and supersymmetry can be broken only on quadruple intersections. Since the nonsupersymmetric case solves the low energy equations of IIB with Reissner-Nordstrom geometry, one might hope to find a conformal quantum mechanical dual for the near horizon geometry. Unfortunately, an analysis [17] reveals that the nonsupersymmetric configuration does not minimize the energy and is expected to be unstable.

By T-dualizing this configuration we obtain $N_{2} N_{3} N_{4}$ intersections of M theory fivebranes on an effective string with $N_{1}$ units of momentum. With the proper normalization of 
charges, the entropy has been calculated macroscopically (2.5) to be $S=2 \pi \sqrt{N_{1} N_{2} N_{3} N_{4}}$, and arguments have been given that this result holds microscopically [9] [10]. By deforming the degenerate fivebranes into a smooth fivebrane, one can use the prescription of [11] to determine the microscopic entropy. One finds that $c_{L}=c_{R}=6 N_{2} N_{3} N_{4}$ up to a negligible correction for large charges, and the entropy agrees with the macroscopic prediction. The left side is almost entirely bosonic, whereas the right side is supersymmetric. Because of the asymmetry of left and right movers, we can view the nonsupersymmetric instability associated with the wrong sign momentum as a tachyon. From the type IIA perspective the momentum is equivalent to $N_{1}$ D-particles whose quantum mechanics we will derive. We expect that this quantum mechanics will apply to any supersymmetric black hole with a Reissner-Nordstrom metric when one ignores corrections based on the transverse six-fold geometry. The full geometry possibly can be incorporated in this quantum mechanics, but the analysis is not within the scope of this paper.

\section{Multistrings at D-Brane Intersections}

In this section we conjecture that the states describing D-particle interactions at the intersections of D-branes are multipronged fundamental strings that attach to the D-particle. In string theory novel states are sometimes required in special compactifications such as the twisted open strings discovered [18] in the context of certain orientifolds. The idea of multipronged strings (multistrings) previously found an application in type IIB string theory to describe certain BPS states [19] 20 including states responsible for exceptional gauge symmetries [21] and nonperturbative states preserving one-quarter of the supersymmetry in $N=4$ Yang-Mills theory [22]. The context in which we are proposing these objects is novel. The considerations of this section are the most conjectural of this paper as we will not at this time try to prove the existence of these objects. Our main argument for invoking these states is that they lead to a quantum mechanical description that satisfies many requirements of a dual to supersymmetric string theory in the background of a Reissner-Nordstrom black hole. Another argument is that we expect the low energy degrees of freedom to carry the charges of all the branes at the intersection. A perhaps more prosaic consideration is that an index theory calculation similar to that of section four was attempted by assuming the presence of the usual D0-D4 matter at these intersections. Not only was the calculation formidably impossible (for me) but also an upper bound on the integral seemed to be too low. By contrast, the calculation with these states is a piece 
of cake and yields the desired result. The natural assumption is that multistrings are the bound (BPS) states of the intersection.

Let us now describe these states. We assume that $n$ D-branes of the same dimension $d$ intersect along some locus such that any two D-branes are orthogonal to each other (can at most intersect in less than $d$ dimensions) and some supersymmetry is preserved. The BPS state that we conjecture has $n+1$ prongs, one end on a D-particle and one end on each of the $n$ branes. Our assumption is that there is always such a string with an endpoint carrying charge $|q|=1$ under the $U(1)$ gauge group of the D-particle. By symmetry each of the $n$ D-branes must contribute $|q|=1 / n$ to this charge. Such a string will have at least three prongs (the case $n=1$ is the usual case) and break the supersymmetry from 32 to no more than 4 supercharges. We then assume that other states for $n \geq 3$ can be obtained by reversing the orientations of an even number of the $n$ strings attached to the $n$ different branes.

We show these states for $n=2$ and $n=3$ in figure one. If the string entering the D-particle carries no charge, the state can be deformed to one that does not interact with the D-particle and should not be considered in the quantum mechanics. Although we have drawn the strings with finite size for clarity, these states are massless and can shrink to a point as appropriate for low energy modes of the quantum mechanics. We assume that the rule requiring the number of orientation changes to be even is related to the fact (2.10) that supersymmetry is broken for an odd number of brane orientation changes for $n \geq 3$. Here we are fixing the D-particle orientation. 


\section{FIGURE 1}

\section{Multistrings At Intersections}

a.
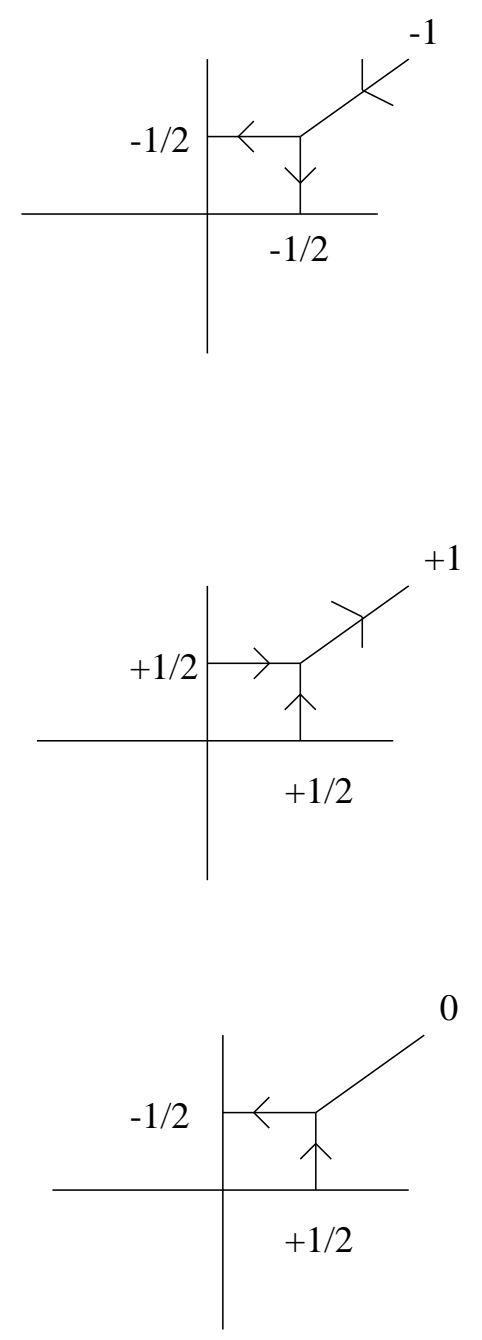

b.
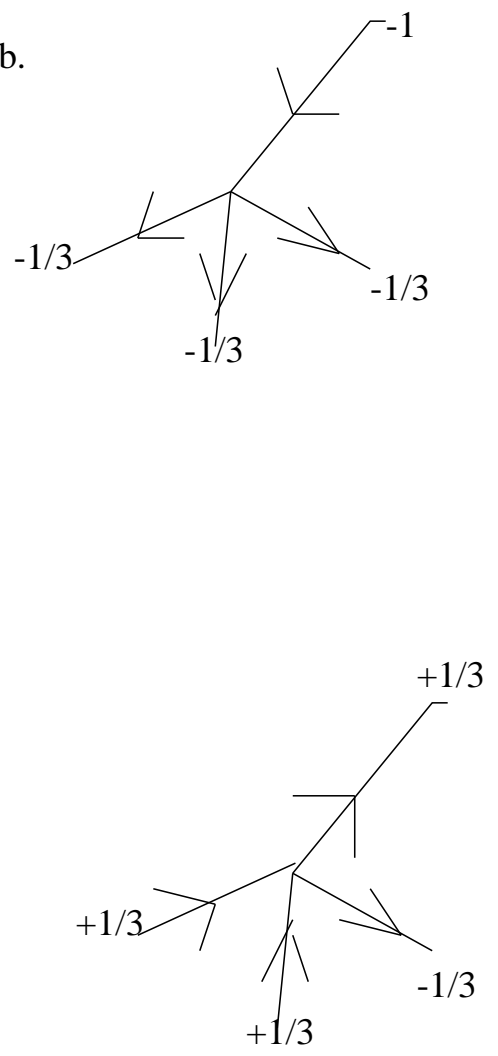

Fig. 1. a. Multistrings localized at an $n=2$ intersection. b. Multistrings at an $n=3$ intersection. There are 3 multistrings with charge $+1 / 3$.

When the intersection of pairs of D-branes has dimension greater than zero, we assume that there is a multistring at the intersection with ends on each of these intersections such that $|q|=2$. Again we can change an even number of the $\frac{n !}{(n-2) ! 2 !}$ orientations to 
obtain other states as shown in figure two for $n=3$. We can iterate this process to higher intersections which are possibly significant for compactifications to less than four dimensions, but in four dimensions the process ends with pairs. Our main consideration here is that the minimal set of objects required by symmetry between the branes is invoked. There are two overall signs both here for the charge and in section four for the index. The overall sign for the charge will not affect the calculation. We are assuming a specific choice of overall sign for the index that yields a result consistent with expectations for the supersymmetric Reissner-Nordstrom black hole.

\section{FIGURE 2}

\section{Multistrings At Intersections Of Intersections}

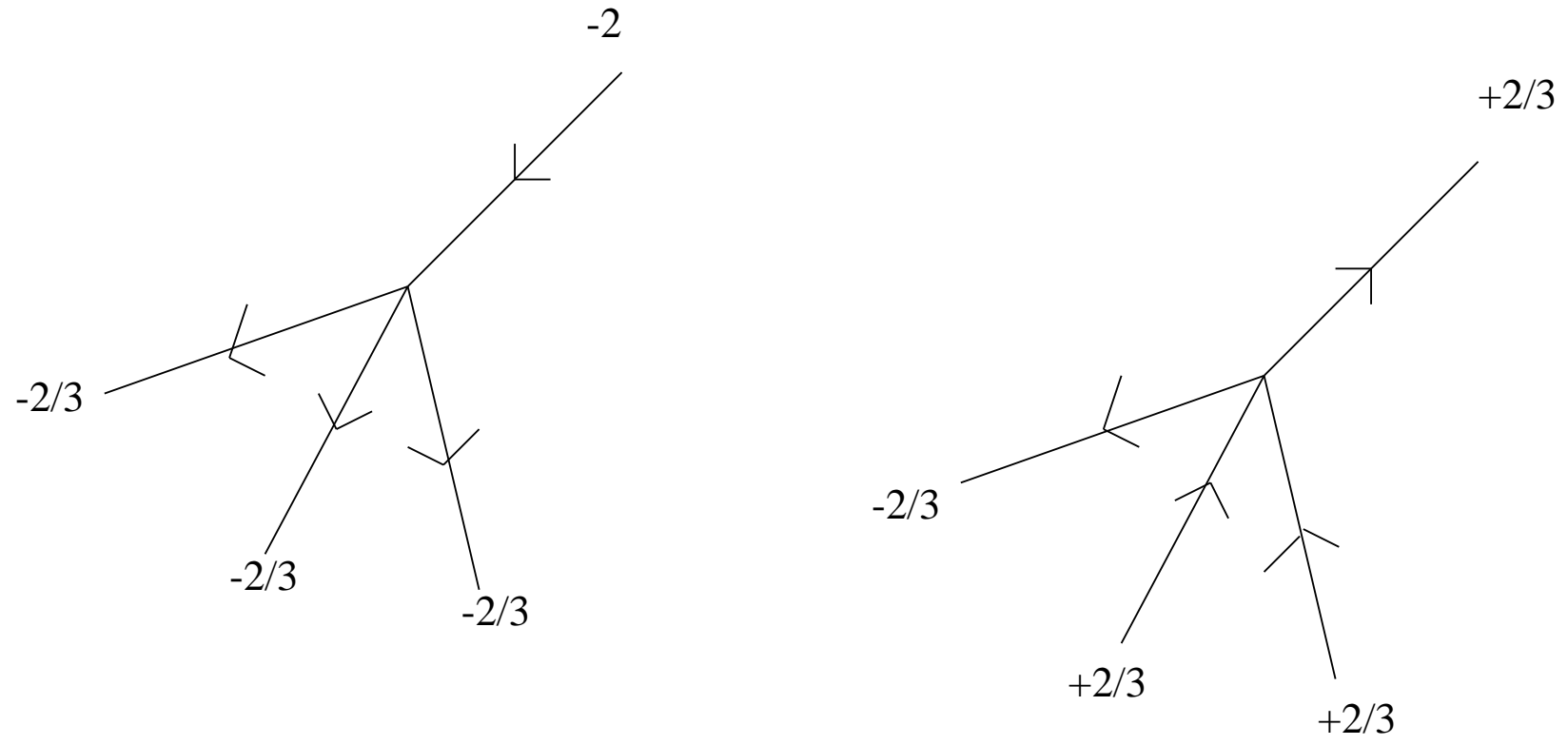

Fig. 2. There are $3+2 / 3$ charged multistrings.

Let us further argue for the plausibility of the multistring states. If one considers two fourbranes intersecting in a membrane, the gauge theory on the membrane is $U(1) \times U(1)$ with hypermultiplets charged concurrently under the two $U(1)$ 's. This theory is the same 
as that for a membrane transverse to a $\mathbf{R}^{4} / \mathbf{Z}_{2}$ orbifold in type IIA. This observation suggests an analogy between intersections of D-branes and orbifolds. If one thinks about the intersection of two fourbranes as a degenerate limit of one smooth fourbrane, the curvature of this fourbrane is not well-defined at the intersection locus. This locus is analogous to the collapsed two-cycle at the $\mathbf{Z}_{2}$ fixed point of the orbifold. At a $\mathbf{Z}_{2}$ fixed point one can obtain half-integer values for the Neveu-Schwarz antisymmetric two-form. Branes which lack moduli to move away from the fixed point can be interpreted as branes with two extra dimensions wrapped around the collapsed two-cycle at the fixed point. The "dimensional" reduction of couplings of the gauge field strength and two-form in the two "extra dimensions" can induce a half-integral charge for the endpoint of a fundamental string on a brane that is stuck at the fixed point. An endpoint that can move away must be integrally charged (the image brane must exist at the fixed point). If a string endpoint does carry half-integral charge, there must be another endpoint stuck at the fixed point carrying half-integral charge by charge conservation.

Interpreting the intersection locus as a fixed locus of an orbifold, we are freezing all moduli that move D-branes of spatial dimension greater than zero away from this locus. (One can also make an analogy between string worldsheet orbifolds (orientifolds) and D-brane worldvolume orbifolds.) Before adding the D-particles there are no fractional charges, and one has the usual theory of the intersection. The D-particles add magnetic flux (the D-particle is an instanton in each of the intersecting branes) to the intersecting fourbranes which is localized at their position. At the intersection there is a $\mathbf{Z}_{2}$ symmetry, exchanging the intersecting branes. The orbifold analogy suggests that the instanton flux due to the D-particle can take half-integral values in each one of the intersecting membranes at the intersection so that the endpoints of the multistring at the intersection carry halfintegral charge. The Ramond-Ramond gauge field on the D-particle couples to the total instanton flux from the intersecting branes. Since the D-particle is a point, the other endpoint of the multistring must provide a cancelling flux. This argument is sensible when all the endpoints are at the intersection. We might expect that massive charged states in the D-particle quantum mechanics do not respect supersymmetry. The analysis of the "Coulomb" branch in section four confirms this expectation. The D-particles must couple to uncharged combinations of multistrings in leaving the intersection.

One can generalize the above remarks to the case of three fourbranes intersecting in a point where the intersection is invariant under $\mathbf{S}_{3}$ permutations of the intersecting branes. Because of this symmetry, it is plausible that the instanton flux of the intersection and 
therefore the charge can be quantized in units of $1 / 3$. The main point is that intersections as a singular limit of smooth D-branes should contain possibly extra massless states localized at the singularity just like what has been found for the other singularities of string theory.

We now come to a crucial distinction between the $n=2$ and $n=3$ cases. A supersymmetric massive deformation of the $n=2$ multistrings along the compact directions is possible since there are two multistrings of opposite charge. The orbifold analogy suggests that the $\mathbf{Z}_{2}$ symmetry of the intersection should be preserved in this deformation. Note that in this deformation only the endpoint attached to the D-particle leaves the intersection. (Figure $1 \mathrm{a}$ is a little misleading.) The $n=2$ case corresponds to two fourbranes intersecting along a two-torus. Assume that brane one is wrapped on $T_{(1)}^{2} \times T_{(2)}^{2}$ while brane two is wrapped on $T_{(1)}^{2} \times T_{(3)}^{2}$. Let the intersection have complex coordinates $y_{2}=y_{3}=0$. There is a BPS deformation direction obtained by requiring $\left|y_{2}\right|=\left|y_{3}\right|$. The mass will be determined by $\left|y_{2}\right|$ so there is an extra $S^{1}$ that decouples in addition to $y_{1}$. This $S^{1}$ degenerates at the intersection which seems to pose a problem for the counting of states. We will show in the next section that this apparent problem does not exist. Note that having obtained this result, the BPS spectrum of the D-particle for the $U(1)$ case is almost identical to that for the D0/D4 bound state problem. The counting of states in the next section will be facilitated by this observation.

Our "rules" give nice results for the counting of states and seem to be logical, but we cannot rule out a different set of states giving equally nice results and being the correct states. If this turns out to be the case, we are consoled by the fact that the "Coulomb" branch of the conformal quantum mechanics (to be derived in section five) should be unchanged. It will be interesting to see whether one can put the existence of these multipronged IIA strings at brane intersections on a firmer footing (perhaps by relating them to $\mathrm{M}$ theory membranes ending on fivebranes).

\section{Bound States at Threshhold and Counting of Microscopic BPS States}

In this section we will calculate the index of supersymmetric ground states in the simplest versions of the theories we have postulated in the previous section. Our result will provide evidence for the formulas we will conjecture for the general case. The calculation will involve bound states at threshhold, and some of the previous relevant work includes [13], 24 33. Our calculations will be similar to the ones given in 27] 28] [29. 


\subsection{Setting up the Calculation}

We will study the case of one D-particle interacting with one intersection of fourbranes having $n=2$ or $n=3$. By our proposal of section three, this theory is a quantum mechanics with four supercharges which can be obtained from the dimensional reduction of the $N=1$ Yang-Mills theory in four dimensions. The formulas of [34] are particularly useful in this regard although we will make some changes in their conventions following [27. Let us first deal with the $n=2$ case. This theory is a $U(1)$ gauge theory with two chiral multiplets having charge $q= \pm 1$. There is also an uncharged chiral multiplet that interacts with the charged multiplets via a superpotential. Additionally, there are some decoupled degrees of freedom. In calculating the index the gauge coupling constant $e$ can be set to any nonzero value as it scales out of the index calculation. For the purpose of this computation we will set $e=2$ in the Lagrangian of [34]. The Lagrangian also depends on another coupling constant $g$ for the superpotential term. Unlike the case of [27] we are considering the dimensional reduction of an $N=1$ not $N=2$ theory so the value of $g$ is not set by supersymmetry. Nevertheless, the calculation of the index cannot depend on this value so long as it is nonzero, and the calculation is simplest when we choose $g=\sqrt{2}$ as in the $N=2$ case. We have argued in section three that $g$ should be nonzero. With these choices the Hamiltonian takes the following form after replacing the nondynamical variables $D$ and $F$ [34] by the values that solve their equations of motion.

$$
\begin{gathered}
H=\frac{1}{2} p^{i} p^{i}+p_{y} p_{y}^{\dagger}+p_{+} p_{+}^{\dagger}+p_{-} p_{-}^{\dagger}+\frac{1}{2}\left(Q_{+} Q_{+}^{\dagger}+Q_{-} Q_{-}^{\dagger}\right)^{2} \\
+\left(x^{i} x^{i}+2 y y^{\dagger}\right)\left(Q_{+} Q_{+}^{\dagger}+Q_{-} Q_{-}^{\dagger}\right)+H_{F} \\
H_{F}=x^{i}\left(M_{+}^{\dagger} \sigma_{i} M_{+}-M_{-}^{\dagger} \sigma_{i} M_{-}\right)+\sqrt{2}\left(y M_{-} u M_{+}-y^{\dagger} M_{-}^{\dagger} u M_{+}^{\dagger}\right) \\
+\sqrt{2}\left(Q_{+} M_{+}^{\dagger} u L^{\dagger}-Q_{+}^{\dagger} M_{+} u L-Q_{-} M_{-}^{\dagger} u L^{\dagger}+Q_{-}^{\dagger} M_{-} u L\right) \\
+\sqrt{2}\left(Q_{-} M_{+} u N+Q_{+} M_{-} u N-Q_{+}^{\dagger} M_{-}^{\dagger} u N^{\dagger}-Q_{-}^{\dagger} M_{+}^{\dagger} u N^{\dagger}\right) \\
i C_{B}=Q_{-}^{\dagger} p_{-}^{\dagger}-Q_{+}^{\dagger} p_{+}^{\dagger}+Q_{+} p_{+}-Q_{-} p_{-} \\
C_{F}=M_{+}^{\dagger} M_{+}-M_{-}^{\dagger} M_{-}
\end{gathered}
$$

where the momenta are $p^{i}=\frac{\delta \mathcal{L}}{\delta \dot{x}^{i}}$, etc. , $x^{i}$ are the spatial components in the reduction of the four-dimensional gauge fields, $\sigma_{i}$ are the usual Pauli matrices, $u=-i \sigma_{2}, y$ is the complex scalar in the neutral chiral multiplet, $Q_{ \pm}$are the complex scalars in the charged chiral multiplets with charges $q= \pm 1$, and $C_{B}, C_{F}$ are the bosonic and fermionic constraints 
generating gauge rotations $\left(\frac{\delta \mathcal{L}}{\delta A_{0}}=C_{B}+C_{F}\right)$. We have used $\dagger$ as hermitian conjugate or complex conjugate depending on the context, and we have chosen the gauge $A_{0}=0$. The complex two-component fermions $L, M_{+}, M_{-}, N$ satisfy the anticommutation relations,

$$
\left\{L_{\alpha}, L_{\beta}^{\dagger}\right\}=\delta_{\alpha \beta} .
$$

Next let us consider the $n=3$ case. This theory is a $U(1)$ gauge theory with three chiral multiplets of charge $+\frac{1}{3}$, three with charge $+\frac{2}{3}$, one with charge -1 , and one having charge -2 . Note that as a four-dimensional theory there would be an anomaly, but this anomaly is irrelevant for the quantum mechanics. A priori we have the possibility of a superpotential coupling together three chiral multiplets of $U(1)$ charges $\frac{1}{3}, \frac{2}{3}$, and -1 . This superpotential could lift some or all of the flat directions of the "Higgs" branch. We will assume here that the superpotential is absent. One reason is that the coupling together of these charges presumably can be deformed into an object not localized at the intersection. Another reason is that the index calculation becomes extremely difficult with a superpotential. Actually, we will have a more concrete statement about a superpotential when we discuss the index calculation. Once we have turned off the superpotential, we are guaranteed by supersymmetry in four dimensions that there will be no perturbative or nonperturbative (for the $\mathrm{U}(1)$ case) corrections. In the quantum mechanics holomorphy should also ensure that this coupling remains zero. The Hamiltonian and constraints for the $n=3$ case then are

$$
\begin{gathered}
H=\frac{1}{2} p^{i} p^{i}+\sum_{m} p_{m} p_{m}^{\dagger}+\frac{1}{2}\left(\sum_{m} q_{m} Q_{m} Q_{m}^{\dagger}\right)^{2} \\
+x^{i} x^{i} \sum_{m} q_{m}^{2} Q_{m} Q_{m}^{\dagger}+H_{F} \\
H_{F}=\sum_{m} q_{m} M_{m}^{\dagger} \sigma \cdot x M_{m}+\sqrt{2}\left(\sum_{m} q_{m} Q_{m} M_{m}^{\dagger} u L^{\dagger}+h . c .\right) \\
i C_{B}=\sum_{m} q_{m} Q_{m} p_{m}-h . c . \\
C_{F}=\sum_{m} q_{m} M_{m}^{\dagger} M_{m}
\end{gathered}
$$

where $m$ indexes the chiral multiplets, $q_{m}$ is the charge, and h.c. is the hermitian conjugate. We can write the supersymmetries as

$$
\begin{aligned}
Q_{\alpha}= & (\sigma \cdot p L)_{\alpha}-\sqrt{2} \sum_{m}\left(u M_{m}^{\dagger}\right)_{\alpha} p_{m}^{\dagger} \\
& +\sqrt{2} i \sum_{m}\left(\sigma \cdot x u M_{m}^{\dagger}\right)_{\alpha} Q_{m} q_{m}-i L_{\alpha} \sum_{m} Q_{m} Q_{m}^{\dagger} q_{m}
\end{aligned}
$$


where

$$
\left\{Q_{\alpha}^{\dagger}, Q_{\beta}\right\}=2 \delta_{\alpha \beta} H-2(\sigma \cdot x)_{\alpha \beta}\left(C_{B}+C_{F}\right)
$$

and

$$
\left\{Q_{\alpha}, Q_{\beta}\right\}=0
$$

We now outline the index calculation. Following [13] the goal is to calculate the supersymmetric index or partition function with the insertion of $(-1)^{F}$,

$$
I=\lim _{\beta \rightarrow \infty} I(\beta)=\lim _{\beta \rightarrow \infty} \operatorname{Tr}(-1)^{F} e^{-\beta H}=n_{B}-n_{F}
$$

This index computes the number of bosons minus the number of fermions in the supersymmetric ground state where $F$ is fermion number. The sum is only over gauge invariant states. If the spectrum were discrete, there would be no dependence on $\beta$. When there is a continuous spectrum above the ground state, the density of bosonic and fermionic states can differ and depend on $\beta$. The usual procedure is to calculate the index as a sum of two terms, $I=I(0)+\Delta I$ where

$$
\Delta I=\int_{0}^{\infty} \frac{d}{d \beta} I(\beta)
$$

The partition function can be constructed perturbatively in powers of $\beta$ so that the $\beta \rightarrow 0$ limit is easily taken. The boundary correction $\Delta I$ is more subtle. The bosonic potential has noncompact (flat) directions along which this potential vanishes. Near these directions the hamiltonian is a supersymmetric harmonic oscillator in the transverse directions. The frequency of the oscillator increases linearly with distance from the origin along a flat direction, but the ground state energy of the supersymmetric oscillator vanishes. One can therefore have finite energy scattering states along these directions so that the index depends on $\beta$, and there is a possible correction $\Delta I$.

One includes a projection onto gauge invariant states $\int_{U(1)} d \theta e^{i \theta C}$ where $C=C_{B}+C_{F}$ so that $I(\beta)$ becomes 27

$$
I(\beta)=\int_{U(1)} d \theta \int d x\left\langle x\left|\operatorname{Tr} e^{i \theta C}(-1)^{F} e^{-\beta H}\right| x\right\rangle=\int_{U(1)} d \theta \int d x\left\langle g x\left|\operatorname{Tr} \Pi(g)(-1)^{F} e^{-\beta H}\right| x\right\rangle
$$

where $x$ denotes the totality of scalar fields, $g(\theta)$ is a gauge transformation, $\Pi(g)=e^{i \theta C_{F}}$, and the volume of $U(1)$ is normalized to unity. Then one obtains

$$
I=\lim _{\beta \rightarrow 0} \int_{U(1)} d \theta \int d x\left\langle g x\left|\operatorname{Tr} \Pi(g)(-1)^{F} e^{-\beta H}\right| x\right\rangle+\Delta I .
$$


It has been shown [27] 29] that the correction or boundary term of the index takes the form

$$
\Delta I=\lim _{R \rightarrow \infty} \int_{|\vec{x}|=R} d x\left\langle g x\left|\frac{x^{i}}{R} \int_{U(1)} \operatorname{Tr} \Psi^{i}(-1)^{F} Q^{\dagger} H^{-1} \Pi(g)\right| x\right\rangle
$$

where $H^{-1}$, the inverse of the Hamiltonian, is defined to be zero on the kernel of $H$, $\Psi^{i}$ is the fermion coefficient of the derivative term in the supercharge $Q$, and $\vec{x}$ is the flat direction with boundary $|\vec{x}|=R$. We will not attempt to rigorously prove that this term vanishes for the cases considered here but instead will argue that it provides a small correction needed to ensure that the index is integral.

\subsection{The Calculation}

The calculation of the index for the $n=2$ case is identical to that presented in [27], and we will not belabor the details. There it was established that the index of supersymmetric ground states is one for the one-dimensional $U(1)$ gauge theory. There are also some zero energy modes decoupled from the gauge theory. These include modes associated with the two directions on the intersection of fourbranes and the zero mode $S^{1}$ discussed in section three. We obtain a total of four fermionic states and four bosonic states for each supersymmetric ground state of the gauge theory. We need to make sure that the degeneracy of the $S^{1}$ at the intersection does not mess up the counting. By cutting off the lower bound on the $y$ integration at $\epsilon$, we can see that there is a vanishing contribution to the principal index $(I(0))$ from the intersection. (There are no inverse powers of $\left|Q_{ \pm}\right|^{2}$ in the integral over the charged scalars in the correction of order $\epsilon$ to this cutoff.) We have also taken $y$ to be noncompact to simplify the index calculation.

There are a couple of new details in the $n=3$ calculation. Fermions from the constraint $C_{F}$ are necessary to saturate the fermion zero modes in $(-1)^{F}$. We consider $C_{F}$ as another component of the $\sigma \cdot x$ term in $H_{F}$ in the exponent. The justification is that the commutator terms from rearrangements are higher order in $\beta$ and vanish in the $\beta \rightarrow 0$ limit as discussed in 28] 29. The integrand in the $x^{i}$ and $\theta$ integrations is then a function of $r^{\prime 2}=x^{i} x^{i}+\theta^{2}$. Let us present the details of this computation. We start with

$$
\lim _{\beta \rightarrow 0} \int d x d Q \int_{-\pi}^{\pi} \frac{d \theta}{2 \pi} \frac{1}{(2 \pi \beta)^{d+3 / 2}} e^{-\sum_{m}\left|e^{i q_{m} \theta} Q_{m}-Q_{m}\right|^{2} / \beta} e^{-\beta V} \operatorname{Tr}\left((-1)^{F} e^{i \theta C_{F}} e^{-\beta H_{F}}\right)
$$


where $V$ is the bosonic potential, $q_{m}$ is the charge of a complex scalar $Q_{m}$, and the trace is over fermions. Rescaling all scalars by $\beta^{-1 / 4}$ yields

$$
\lim _{\beta \rightarrow 0} \int d x d Q \int_{-\pi}^{\pi} \frac{d \theta}{2 \pi} \frac{\beta^{-d / 2-3 / 4}}{(2 \pi \beta)^{d+3 / 2}} e^{-\sum_{m}\left|e^{i q_{m} \theta} Q_{m}-Q_{m}\right|^{2} / \beta^{3 / 2}} e^{-V} \operatorname{Tr}\left((-1)^{F} e^{i \theta C_{F}} e^{-\beta^{3 / 4} H_{F}}\right) .
$$

Expanding the $\sum_{m}\left|e^{i q_{m} \theta} Q_{m}-Q_{m}\right|^{2} / \beta^{3 / 2}$ and taking into account the smallness of $\beta$ gives

$$
\lim _{\beta \rightarrow 0} \int d x d Q \int_{-\pi}^{\pi} \frac{d \theta}{2 \pi} \frac{\beta^{-d / 2-3 / 4}}{(2 \pi \beta)^{d+3 / 2}} e^{-\theta^{2} \sum_{m} q_{m}^{2}\left|Q_{m}\right|^{2} / \beta^{3 / 2}} e^{-V} \operatorname{Tr}\left((-1)^{F} e^{i \theta C_{F}} e^{-\beta^{3 / 4} H_{F}}\right)
$$

We might worry that higher order terms in $\theta$ should be included for the $q_{8}=-2$ term. We have explicitly verified by a change of variables for $\theta$ to extract the contribution near $\theta= \pm \pi$ that this contribution vanishes. We then rescale $\theta$ by $\beta^{3 / 4}$ and combine $C_{F}$ with $H_{F}$ to get

$$
\lim _{\beta \rightarrow 0} \int d x d Q \int_{-\infty}^{\infty} \frac{d \theta}{2 \pi} \frac{\beta^{-d / 2}}{(2 \pi \beta)^{d+3 / 2}} e^{-\theta^{2} \sum_{m} q_{m}^{2}\left|Q_{m}\right|^{2}} e^{-V} \operatorname{Tr}\left((-1)^{F} e^{-\beta^{3 / 4}\left(H_{F}+i \theta C_{F}\right)}\right) .
$$

Now by including the appropriate number of fermions, we find that the $\beta$ dependence has disappeared,

$$
\int d x d Q \int_{-\infty}^{\infty} \frac{d \theta}{2 \pi} \frac{e^{-\left(\theta^{2}+r^{2}\right) \sum_{m} q_{m}^{2}\left|Q_{m}\right|^{2}}}{(2 \pi)^{d+3 / 2}} e^{-\frac{1}{2}\left(\sum_{m} q_{m}\left|Q_{m}\right|^{2}\right)^{2}} \frac{1}{(2 d+2) !} \operatorname{Tr}\left((-1)^{F}\left(H_{F}+i \theta C_{F}\right)^{2 d+2}\right) .
$$

After a calculation we obtain for the fermionic trace the result

$$
4\left(\vec{x}^{2}+\theta^{2}\right)^{d-1} \prod_{m} q_{m}^{2}(2 d+2) !\left(\sum_{m} q_{m}\left|Q_{m}\right|^{2}\right)^{2}
$$

where there is an ambiguity for the overall sign that we are taking to be positive as discussed in section three. Combining the $x^{i}$ and $\theta$ integrations gives

$$
4 \prod_{m} q_{m}^{2} \int_{0}^{\infty} d r^{\prime} \frac{r^{\prime 2 d+1} e^{-r^{\prime 2} \sum_{m} q_{m}^{2}\left|Q_{m}\right|^{2}}}{(2 \pi)(2 \pi)^{d+3 / 2}} \int_{S^{3}} d \Omega_{3} \int d Q\left(\sum_{m} q_{m}\left|Q_{m}\right|^{2}\right)^{2} e^{\frac{-1}{2}\left(\sum_{m} q_{m}\left|Q_{m}\right|^{2}\right)^{2}}
$$

We get

$$
\frac{4 \prod_{m} q_{m}^{2} \Gamma(d+1) \pi^{2}}{(2 \pi)^{(d+3 / 2)+1}} \int d Q \frac{\left(\sum_{m} q_{m}\left|Q_{m}\right|^{2}\right)^{2}}{\left(\sum_{m} q_{m}^{2}\left|Q_{m}\right|^{2}\right)^{d+1}} e^{\frac{-1}{2}\left(\sum_{m} q_{m}\left|Q_{m}\right|^{2}\right)^{2}}
$$


The remaining integration is done by substituting $Q_{m}=\frac{1}{\sqrt{2}}\left(Q_{m r}+i Q_{m i}\right)$ and rescaling $Q_{m}$ by $\frac{1}{q_{m}}$ so that the integration is over real $Q_{m r}, Q_{m i}$, and the $\prod_{m} q_{m}^{2}$ factor disappears. The remaining computation is straightforward.

We used the computer program Vegas written by G.P. Lepage. Our computation involved $10^{5}$ integrand evaluations per iteration and 10 iterations. We obtained the result for the $n=3$ case,

$$
I(0)=6.0097 \pm .0053 .
$$

Given that the principal contribution is very close to 6 , we expect a negligible boundary contribution. There are two flat directions, $Q_{m}=0$ all $m$ or $x^{i}=0$ and $\sum_{m} q_{m} Q_{m} Q_{m}^{\dagger}=0$. An intuitive argument for ignoring the "Coulomb" boundary term is that the charged multiplets become very heavy along this direction leaving a free $U(1)$ theory. Unlike the case of [28] [29] we are not starting from a nonabelian theory so there is no left over Weyl invariance, and the boundary term should be [28] the negative of the principal term for $U(1)$ which vanishes. Without a superpotential, we cannot ignore the "Higgs" boundary.

We will follow somewhat the analysis presented in [29] to determine the asymptotic Hamiltonian in the flat directions for the ground state of the massive modes. To this level of approximation, we will show that the boundary term is appropriately small. We will not "prove" that the total index is 6 .

Let us look at the "Coulomb" direction. By a unitary transformation of the $M_{m}$ fermions we can write the first term of $H_{F}$ as

$$
r \sum_{m} q_{m}\left(M_{m 1}^{\dagger} M_{m 1}-M_{m 2}^{\dagger} M_{m 2}\right)
$$

where $r=\sqrt{x^{i} x^{i}}$. We decompose the ground state wave function as

$$
\Psi=\Psi_{H O} \Psi_{F l a t}\left(x^{i}\right)|F\rangle
$$

where

$$
\Psi_{H O}=\prod_{m}\left(\frac{r}{\left|q_{m}\right| \pi}\right)^{\frac{1}{4}} e^{-r \sum_{m}\left|Q_{m}\right|^{2}\left|q_{m}\right|}
$$

and

$$
|F\rangle=\prod_{m} M_{m \alpha_{m}}^{\dagger}|0\rangle
$$


where $\alpha_{m}=\frac{1}{2}\left(3+\frac{q_{m}}{\left|q_{m}\right|}\right)$. Thus, the supersymmetric harmonic oscillator part of the Hamiltonian vanishes on these states. Next we add up the other contributions. It is convenient to use harmonic oscillator operators

$$
\begin{aligned}
Q_{m a} & =\frac{1}{\sqrt{2 r\left|q_{m}\right|}}\left(a_{m a}+a_{m a}^{\dagger}\right) \\
\partial_{Q_{m a}} & =\sqrt{\frac{r\left|q_{m}\right|}{2}}\left(a_{m a}-a_{m a}^{\dagger}\right)
\end{aligned}
$$

with $Q_{m}=\frac{1}{\sqrt{2}}\left(Q_{m r}+i Q_{m i}\right)$ and $\left[a_{m a}, a_{n b}^{\dagger}\right]=\delta_{a b} \delta_{m n}$. Note that for $\Psi_{\text {Flat }}=1,\left\langle\Psi\left|\partial_{r}\right| \Psi\right\rangle=$ 0 . The net result is that

$$
H_{\text {Flat }}=-\frac{1}{2} \Delta_{x}+\frac{d(d+2)}{8 r^{2}}-\frac{d_{+} d_{-}}{2 r^{2}}
$$

where $d$ is the number of chiral multiplets, $d_{+}$the number of positively charged ones, and $d_{-}$the number of negatively charged ones. We follow [29 in realizing that we can lower the ground state energy by a redefinition

$$
\left|F^{\prime}\right\rangle=\left(1-\frac{1}{\sqrt{2} r} \sum_{m} \frac{q_{m}}{\left|q_{m}\right|} Q_{m} M_{m}^{\dagger} u L^{\dagger}\right)|F\rangle .
$$

We then have

$$
H_{\text {Flat }}=-\frac{1}{2} \Delta_{x}+\frac{d(d-2)}{8 r^{2}}-\frac{d_{+} d_{-}}{2 r^{2}},
$$

ignoring terms of lower order in $1 / r$. For our case $d_{+}=6, d_{-}=2, H_{\text {Flat }}=-\frac{1}{2} \Delta_{x}$, and the $U(1)$ argument 28] for a vanishing correction is a good one.

To analyze the "Higgs" boundary we first choose a gauge in which $Q_{1}$ is real. Then we make the following change of coordinates:

$$
\begin{aligned}
& Q_{1}, Q_{m} \longrightarrow x^{0}, Q_{m}^{\prime} \\
& x^{0}=\frac{\sum_{m} q_{m}\left|Q_{m}\right|^{2}}{\sqrt{2 \sum_{m} q_{m}^{2}\left|Q_{m}\right|^{2}}} . \\
& Q_{m}^{\prime}=Q_{m}, \quad m>1
\end{aligned}
$$

The "Higgs" branch corresponds to $x^{i}=x^{0}=0$, and the boundary corresponds to $\nu \rightarrow \infty$ with $\nu=\sqrt{2 \sum_{m} q_{m}^{2}\left|Q_{m}\right|^{2}}$. Under the change of variables

$$
\begin{aligned}
\sum_{m} p_{m} p_{m}^{\dagger} & =\left(\frac{1}{2}-\frac{2 x^{0}}{\nu^{3}} \sum_{m} q_{m}^{3}\left|Q_{m}\right|^{2}+\frac{x^{0^{2}}}{\nu^{4}} \sum_{m} q_{m}^{4}\left|Q_{m}\right|^{2}\right) p^{0} p^{0} \\
& -i\left(\frac{\sum_{m} q_{m}}{\nu}-\frac{2}{\nu^{3}} \sum_{m} q_{m}^{3}\left|Q_{m}\right|^{2}-\frac{x^{0}}{\nu^{2}} \sum_{m} q_{m}^{2}+\frac{3 x^{0}}{\nu^{4}} \sum_{m} q_{m}^{4}\left|Q_{m}\right|^{2}\right) p^{0} \\
& +2\left[\sum_{m}\left(\frac{q_{m}}{\nu}-\frac{q_{m}^{2} x^{0}}{\nu^{2}}\right)\left(Q_{m}^{\prime} p_{m}^{\prime}+Q_{m}^{\prime \dagger} p_{m}^{\prime \dagger}\right)\right] p^{0}+\sum_{m} p_{m}^{\prime} p_{m}^{\prime \dagger}
\end{aligned}
$$


Note that $\sum_{m} q_{m}=0$ here. We have not converted all the sums to primed variables. To lowest order in $x^{0}$ and $1 / \nu$, the bosonic Hamiltonian in the massive directions becomes

$$
H_{H O}=\frac{1}{2}\left(p^{i} p^{i}+p^{0} p^{0}\right)+\frac{1}{2} \nu^{2}\left(Q_{m}^{\prime}\right)\left(x^{i} x^{i}+x^{0} x^{0}\right)
$$

Again we decompose the ground state as

$$
\Psi=\Psi_{H O} \Psi_{\text {Flat }}\left(Q_{m}^{\prime}\right)|F\rangle
$$

where

$$
\Psi_{H O}=\frac{\nu\left(Q_{m}^{\prime}\right)}{\pi} e^{-\frac{\nu x^{\mu} x^{\mu}}{2}}
$$

and

$$
|F\rangle=\frac{1}{2}\left[1-\frac{\sqrt{2}}{\nu} \sum_{m} q_{m} Q_{m}^{\prime} M_{m}^{\dagger} u L^{\dagger}+\frac{1}{\nu^{2}}\left(\sum_{m} q_{m} Q_{m}^{\prime} M_{m}^{\dagger} u L^{\dagger}\right)^{2}\right]|0\rangle .
$$

Unprimed sums are converted to primed sums by the substitution

$$
Q_{1}^{2}=-\frac{1}{q_{1}} \sum_{m} q_{m}\left|Q_{m}^{\prime}\right|^{2}
$$

to zeroth order in $x^{0}$. By supersymmetry $M_{1}$ is no longer an independent fermion on the "Higgs" branch. We find that $\left(H_{H O}+H_{F}^{2}\right) \Psi=0$ where $H_{F}^{2}$ is the term of $H_{F}$ depending on the $Q_{m}^{\prime}\left(x^{0}=0\right)$. Also, the $\sigma \cdot x$ term of $H_{F}$ gives zero contribution $\left(\left\langle\Psi\left|H_{F}^{1}\right| \Psi\right\rangle=0\right)$. There are many other contributions of order $\frac{1}{\nu^{2}}$ from the Hamiltonian. In addition to the terms in the change of variables, the Hamiltonian contains some other correction terms, and we find

$$
\begin{aligned}
& \Delta H=q_{1}^{2} x^{0} x^{0} x^{\mu} x^{\mu}-\sqrt{2}\left(\frac{1}{2} \frac{x^{0^{2}}}{Q_{1}}-\frac{1}{8} \frac{\nu^{2} x^{0^{2}}}{q_{1}^{2} Q_{1}^{3}}\right) q_{1}\left(M_{1}^{\dagger} u L^{\dagger}-M_{1} u L\right) \\
& +i\left(\frac{x^{0}}{\nu^{2}} \sum_{m} q_{m}^{2}+\frac{3 x^{0}}{\nu^{4}} \sum_{m}\left(q_{m}^{4}-q_{1}^{3} q_{m}\right)\left|Q_{m}^{\prime}\right|^{2}\right) p^{0} \\
& -\frac{2 x^{0}}{\nu^{2}} \sum_{m} q_{m}^{2}\left(Q_{m}^{\prime} p_{m}^{\prime} p^{0}+{Q_{m}^{\prime}}^{\dagger} p_{m}^{\prime}{ }^{\dagger} p^{0}\right)+\sum_{m} p_{m}^{\prime} p_{m}^{\prime}{ }^{\dagger}
\end{aligned}
$$

where we have ignored vanishing or lower order terms. After some calculation we have obtained the following result for $H_{\text {Flat }}$,

$$
\begin{aligned}
H_{\text {Flat }} & =-\frac{1}{2} \Delta_{Q_{m}^{\prime}}+\frac{1}{\nu^{4}}\left[\frac{1}{162}\left(\left|Q_{2}^{\prime}\right|^{2}+\left|Q_{3}^{\prime}\right|^{2}\right)\right. \\
& \left.+\frac{17}{18}\left(\left|Q_{4}^{\prime}\right|^{2}+\left|Q_{5}^{\prime}\right|^{2}+\left|Q_{6}^{\prime}\right|^{2}\right)+\frac{67}{18}\left|Q_{7}^{\prime}\right|^{2}+\frac{55}{6}\left|Q_{8}^{\prime}\right|^{2}\right]
\end{aligned}
$$


where $q_{2}=q_{3}=1 / 3, q_{4}=q_{5}=q_{6}=2 / 3, q_{7}=-1$, and $q_{8}=-2$. On the other hand,

$$
\nu^{2}=\frac{4}{9}\left(\left|Q_{4}^{\prime}\right|^{2}+\left|Q_{5}^{\prime}\right|^{2}+\left|Q_{6}^{\prime}\right|^{2}\right)+\frac{8}{3}\left|Q_{7}^{\prime}\right|^{2}+\frac{28}{3}\left|Q_{8}^{\prime}\right|^{2} .
$$

The constraint that $Q_{1}^{2} \geq 0$ implies that no $Q_{m}^{\prime}$ can have order greater than $\nu$. Thus, the term in brackets has order $\frac{1}{\nu^{2}}$. We note that the potential $V\left(Q^{\prime}\right)>\frac{7 \times 10^{-5}}{r^{2}}$ where $r=\sqrt{\sum_{m}\left|Q_{m}^{\prime}\right|^{2}}$.

The calculation of the boundary term is complicated by this correction as well as the constraint that $Q_{1}^{2} \geq 0$. Taking $H_{\text {Flat }}$ to be the free Laplacian, we apply again the argument of [28], 1]

$$
\begin{aligned}
\Delta I & =-\lim _{\beta \rightarrow 0} \int d Q^{\prime} \int_{-\pi}^{\pi} \frac{d \theta}{2 \pi} \frac{1}{(2 \pi \beta)^{7}} e^{-\sum_{m}\left|e^{i q_{m} \theta} Q_{m}^{\prime}-Q_{m}^{\prime}\right|^{2} / \beta} \operatorname{Tr}\left((-1)^{F} e^{i \theta\left(\sum_{m} q_{m} M_{m}^{\dagger} M_{m}\right)}\right) \\
& = \pm 2 \int d Q^{\prime} \int_{0}^{\pi} \frac{d \theta}{(2 \pi)^{8}} e^{-\sum_{m}\left|e^{i q_{m} \theta} Q_{m}^{\prime}-Q_{m}^{\prime}\right|^{2}} \prod_{m} \sin ^{2}\left(\theta q_{m}\right) \\
& = \pm \frac{1}{2^{7} \pi} \int_{0}^{\pi} d \theta \prod_{m} \frac{\sin ^{2}\left(\theta q_{m}\right)}{\left(1-\cos _{\left.\left(\theta q_{m}\right)\right)}\right.} \\
& = \pm \frac{1}{\pi} \int_{0}^{\pi} d \theta \cos ^{2}(\theta) \cos ^{2}\left(\frac{\theta}{2}\right) \cos ^{6}\left(\frac{\theta}{3}\right) \cos ^{4}\left(\frac{\theta}{6}\right)
\end{aligned}
$$

The correction computed this way turns out to be

$$
\Delta I= \pm 0.2104
$$

The potential is positive definite and can only decrease the boundary contribution given that there are no tachyons in this theory. Also, the constraint that $Q_{1}^{2} \geq 0$ significantly decreases the boundary contribution. Given that the total index is integral and our approximations, we have provided strong evidence that

$$
I_{n=3}=I(0)+\Delta I=6 .
$$

We also note that no other integer is consistent with these results. There is a $\mathbf{Z}_{6}$ singularity along $Q_{8}^{\prime}=r$, and the $U(1)=S^{1}$ becomes an $R P^{1}$ there. This situation makes the direct boundary calculation more difficult. This singularity does not contribute to the $\beta \rightarrow 0$ calculations. Had we chosen coordinates with $Q_{8} \rightarrow x^{0}$, we might have avoided this problem. We are somewhat baffled by the role of this singularity in the "Higgs" branch.

1 I am grateful to A. Konechny for reminding me of this argument. 
We have recalculated the boundary term by interchanging $Q_{1}$ and $Q_{8}$, neglecting the asymptotic potential. We need to compute

$$
\begin{aligned}
& \Delta I= \pm \frac{1}{\operatorname{Volume}\left(S^{13}\right) 2 \pi \cdot 2^{13}} \lim _{R \rightarrow \infty} \int_{-\pi}^{\pi} d \theta \int_{r=R, Q_{8}^{2} \geq 0} \prod_{m} d Q_{m}^{\prime} \frac{1}{\sqrt{\sum_{m}\left|Q_{m}^{\prime}\right|^{2}}} . \\
& \quad \frac{\operatorname{Tr}\left((-1)^{F} e^{i \theta\left(\sum_{m} q_{m} M_{m}^{\dagger} M_{m}\right)}\right)}{\left(\sum_{m}\left|Q_{m}^{\prime}\right|^{2}\left(1-\cos \left(\theta q_{m}\right)\right)\right)^{6}}
\end{aligned}
$$

Using Vegas we obtain the result

$$
\Delta I= \pm 0.03270 \pm .00004
$$

As expected this result is significantly lower than (4.46), and we expect a further decrease by properly determining the propagator.

A possible heuristic argument for the "Higgs" boundary term being negligible is as follows. Suppose we introduce a superpotential with infinitesimal gauge invariant cubic and quartic couplings. Including the $D$ term there are enough constraints on the eight chiral multiplets to lift all of the flat directions so that the "Higgs" branch is massive and introduces no boundary correction. Does this superpotential change the principal index? Since these couplings only multiply the superpotential terms, we cannot do a universal rescaling to make them large. These couplings have the dimension of mass to some power and are negligible in the high temperature $(\beta \rightarrow 0)$ limit. Also, the limit that the couplings vanish does not produce a singularity in the principal index calculation. In conclusion, we argue that the boundary correction from the "Higgs" branch produces a very small correction in the principal index so that the index is integral.

\subsection{Counting BPS States}

In the last section we have calculated the index of supersymmetric ground states in the simplest examples of the $n=2$ and $n=3$ theories. We will now use this result along with some plausible assumptions to count the BPS ground states and determine the entropy. Our first assumption is that the index actually counts the ground states in these theories. In any case it counts states that will remain massless under smooth deformations of the theory. The degeneracy of states will be bounded from below by the degeneracies determined from the index. We have seen in section two that the Reissner-Nordstrom metric is asymptotically flat. At large distances from the intersections, the D-particles experience flat ten-dimensional spacetime. We therefore assume that there is a unique 
bound state of $N$ D-particles for every $N$ (as has apparently been shown as an index [33]). Our final assumption is that the D-particles and their bound states can interact with any of the $N_{1} N_{5}$ intersections for $n=2$ or $N_{2} N_{3} N_{4}$ intersections for $n=3$ to form the same number of bound states that we have obtained in the one intersection case.

The $n=2$ index calculation indicates that there are four massless bosonic modes and four massless fermionic modes for a D-particle interacting with one intersection. With the above assumptions we write down the following generating function for the degeneracy of $N_{0}$ D-particles interacting with $N_{1} N_{5}$ intersections,

$$
Z=\prod_{n} \frac{\left(1+q^{n}\right)^{4 N_{1} N_{5}}}{\left(1-q^{n}\right)^{4 N_{1} N_{5}}}=\sum_{N_{0}} d\left(N_{0}\right) q^{N_{0}}
$$

In the above product $n$ indexes the number of D-particles that are bound together. The maximum $n$ is $N_{0}$. For large $N_{0}$ this formula implies $d\left(N_{0}\right) \sim \exp \left(2 \pi \sqrt{N_{0} N_{1} N_{5}}\right)$, exactly the result obtained from the onebrane-fivebrane system in previous calculations [4] [15] [16].

The $n=3$ calculation reveals six massless bosonic modes for a D-particle interacting with one intersection. Using our assumptions we determine the generating formula for the degeneracy of $N_{1}$ D-particles interacting with $N_{2} N_{3} N_{4}$ intersections to be

$$
Z=\prod_{n}\left(1-q^{n}\right)^{-6 N_{2} N_{3} N_{4}}=\sum_{N_{1}} d\left(N_{1}\right) q^{N_{1}}
$$

The maximum $n$ is $N_{1}$. Again, we have the previously determined result that $d\left(N_{1}\right) \sim$ $\exp \left(2 \pi \sqrt{N_{1} N_{2} N_{3} N_{4}}\right)$ [9] [11]. Now that we have a little confidence in our theories, we will see in the next section what they imply for the quantum mechanics of four-dimensional black holes.

\section{The Quantum Mechanical System}

\subsection{Generalities}

Let us first write down Lagrangians for the $n=2$ and $n=3$ theories following from dimensional reduction of four-dimensional theories [34]. Note again that the $n=3$ theories are the dimensional reduction of anomalous four-dimensional theories.

For the $n=2$ case we will again assume the superpotential is that given by $N=2$ supersymmetry in four dimensions (although this is not essential). We denote neutral scalars in the adjoint of $U\left(N_{0}\right)$ by $Z_{\mu}=Z_{\mu}^{a} T^{a}$ and charged scalars by $A_{\alpha \beta \gamma}, \bar{A}^{\alpha \beta \gamma}, B^{\alpha \beta \gamma}$, 
and $\bar{B}_{\alpha \beta \gamma}$ where the first (gauge) index runs from 1 to $N_{0}$, the second has $N_{1}$ values, and the third $N_{5}$ values. We single out the three components of the four-dimensional gauge field in the dimensional reduction as $x_{i}$. The superpartners of the charged scalars are $\psi_{A}$ and $\psi_{B}$ while those of the neutral scalars are $\lambda_{L}$ and $\lambda_{N}$. Our hermitian generators $\left(T^{a}\right)_{\alpha}{ }^{\beta}$ of $U\left(N_{0}\right)$ satisfy $\left[T^{a}, T^{b}\right]=i f^{a b c} T^{c}$ and $\operatorname{tr}\left(T^{a} T^{b}\right)=\frac{1}{2} \delta^{a b}$ where $f^{a b c}$ are the structure constants of $S U\left(N_{0}\right)$ (The $U(1)$ generator is $\left(T^{N_{0}}\right)_{\alpha}{ }^{\beta}=\frac{1}{\sqrt{2 N_{0}}} \delta_{\alpha}{ }^{\beta}$.).

The Lagrangian is written as follows in the $A_{0}=0$ gauge.

$$
\begin{aligned}
\mathcal{L}_{n=2} & =\frac{1}{2} \dot{Z}_{\mu}^{a} \dot{Z}_{\mu}^{a}+\dot{\bar{A}} \dot{A}+\dot{\bar{B}} \dot{B}-g^{2} \operatorname{tr}\left(\left[Z_{\mu}, Z_{\nu}\right]^{2}\right) \\
& -g^{2}\left(\left|Z_{\mu} A\right|^{2}+\left|Z_{\mu} \bar{B}\right|^{2}\right)-\frac{g^{2}}{2}\left(\bar{A} T^{a} A-B T^{a} \bar{B}\right)^{2}-2 g^{2}\left|B T^{a} A\right|^{2} \\
& +i \bar{\lambda}_{L}^{a} \dot{\lambda}_{L}^{a}+i \bar{\lambda}_{N}^{a} \dot{\lambda}_{N}^{a}+i \bar{\psi}_{A} \dot{\psi}_{A}+i \bar{\psi}_{B} \dot{\psi}_{B} \\
& -g \bar{\lambda}_{L}^{a}\left[\sigma \cdot x, \lambda_{L}\right]^{a}-g \bar{\lambda}_{N}^{a}\left[\sigma \cdot x, \lambda_{N}\right]^{a}-g \bar{\psi}_{A} \sigma \cdot x \psi_{A}+g \bar{\psi}_{B} \sigma \cdot x^{T} \psi_{B} \\
& -\sqrt{2} g\left(\psi_{B} u y \psi_{A}-\bar{\psi}_{A} u \bar{y} \bar{\psi}_{B}\right) \\
& +\sqrt{2} g\left(\bar{A} T^{a} \psi_{A} u \lambda_{L}^{a}-\bar{\lambda}_{L}^{a} u \bar{\psi}_{A} T^{a} A+B T^{a} \bar{\psi}_{B} u \bar{\lambda}_{L}^{a}-\lambda_{L}^{a} u \psi_{B} T^{a} \bar{B}\right) \\
& +\sqrt{2} g\left(\bar{A} T^{a} \bar{\lambda}_{N}^{a} u \bar{\psi}_{B}-\psi_{B} u \lambda_{N}^{a} T^{a} A-B T^{a} \lambda_{N}^{a} u \psi_{A}+\bar{\psi}_{A} u \bar{\lambda}_{N}^{a} T^{a} \bar{B}\right)
\end{aligned}
$$

where $y, \bar{y}$ are the components of $Z_{\mu}$ transverse to $x_{i}$, and we have suppressed most of the indices. The $a$ and $\mu$ indices should be summed over. The Gauss' law constraints are

$$
\begin{aligned}
G_{n=2}^{a} & =-2 i g\left[Z_{\mu}, \dot{Z}_{\mu}\right]^{a}+i g \frac{d}{d t}\left(\bar{A} T^{a} A-B T^{a} \bar{B}\right) \\
& -2 g\left[\bar{\lambda}_{L}, \lambda_{L}\right]^{a}-2 g\left[\bar{\lambda}_{N}, \lambda_{N}\right]^{a}-g \bar{\psi}_{A} T^{a} \psi_{A}+g \bar{\psi}_{B} T^{a T} \psi_{B}
\end{aligned} .
$$

The $n=3$ Lagrangian is a little easier to write. We are not including superpotentials in our analysis here though they may be significant at higher energies. The charged scalars are $Q_{m \alpha \beta \gamma}{ }^{\delta}, R_{n}{ }^{\alpha \beta \gamma \delta}$ and hermitian conjugates where the first $(\alpha)$ index is a $U\left(N_{1}\right)$ gauge index and the $\beta, \gamma$, and $\delta$ indices are indices for the fundamental representations of $U\left(N_{2}\right)$, $U\left(N_{3}\right)$, and $U\left(N_{4}\right) ; m$ runs from 1 to 6 where the upper (dual) index can be any of the 
last three indices; and $n$ runs from 7 to 8 . The charged superpartners are $\psi_{m}$ and $\psi_{n}$.

$$
\begin{aligned}
\mathcal{L}_{n=3} & =\frac{1}{2} \sum_{a} \dot{x}_{i}^{a} \dot{x}_{i}^{a}+\sum_{m} \dot{\bar{Q}}_{m} \dot{Q}_{m}+\sum_{n} \dot{\bar{R}}_{n} \dot{R}_{n}-g^{2} \operatorname{tr}\left(\left[x_{i}, x_{j}\right]^{2}\right) \\
& -g^{2}\left(\sum_{m, a} q_{m}^{a}{ }^{2}\left|x_{i}^{a} T^{a} Q_{m}\right|^{2}+\sum_{n, a} q_{n}^{a}{ }^{2}\left|R_{n} x_{i}^{a} T^{a}\right|^{2}\right)-\frac{g^{2}}{2}\left(\sum_{m, a} q_{m}^{a} \bar{Q}_{m} T^{a} Q_{m}+\sum_{n, a} q_{n}^{a} R_{n} T^{a} \bar{R}_{n}\right)^{2} \\
& +i \sum_{a} \bar{\lambda}_{L}^{a} \dot{\lambda}_{L}^{a}+i \sum_{m} \bar{\psi}_{m} \dot{\psi}_{m}+i \sum_{n} \bar{\psi}_{n} \dot{\psi}_{n} \\
& -g \sum_{a} \bar{\lambda}_{L}^{a}\left[\sigma \cdot x, \lambda_{L}\right]^{a}-g \sum_{m, a} q_{m}^{a} \bar{\psi}_{m} \sigma \cdot x^{a} T^{a} \psi_{m}-g \sum_{n, a} q_{n}^{a} \bar{\psi}_{n} \sigma \cdot x^{a} T^{a T} \psi_{n} \\
& +\sqrt{2} g\left[\sum_{m, a} q_{m}^{a}\left(\bar{Q}_{m} T^{a} \psi_{m} u \lambda_{L}^{a}-\bar{\lambda}_{L}^{a} u \bar{\psi}_{m} T^{a} Q_{m}\right)-\sum_{n, a} q_{n}^{a}\left(R_{n} T^{a} \bar{\psi}_{n} u \bar{\lambda}_{L}^{a}-\lambda_{L}^{a} u \psi_{n} T^{a} \bar{R}_{n}\right)\right]
\end{aligned}
$$

where the $q_{r}^{a}=\frac{q_{r}}{\left|q_{r}\right|}$ for $a \neq N_{1}, q_{r}^{N_{1}}=q_{r}$ with $r=m$ or $n$, and the $q_{m}, q_{n}$ have been previously given in section four. The Gauss' law constraints are

$$
\begin{aligned}
G_{n=3}^{a} & =-2 i g\left[x_{i}, \dot{x}_{i}\right]^{a}+i g \frac{d}{d t}\left(\sum_{m} q_{m}^{a} \bar{Q}_{m} T^{a} Q_{m}+\sum_{n} q_{n}^{a} R_{n} T^{a} \bar{R}_{n}\right) \\
& -2 g\left[\bar{\lambda}_{L}, \lambda_{L}\right]^{a}-g\left(\sum_{m} q_{m}^{a} \bar{\psi}_{m} T^{a} \psi_{m}+\sum_{n} q_{n}^{a} \bar{\psi}_{n} T^{a T} \psi_{n}\right)
\end{aligned} .
$$

The supersymmetries of this action are

$$
\begin{aligned}
\delta_{\eta} x_{i}^{a} & =i \bar{\lambda}^{a} \sigma_{i} \eta-i \bar{\eta} \sigma_{i} \lambda^{a} \\
\delta_{\eta} \lambda^{a} & =\frac{i}{2} g \sigma_{i j}\left[x_{i}, x_{j}\right]^{a} \eta-i g\left(\sum_{m} q_{m}^{a} \bar{Q}_{m} T^{a} Q_{m}+\sum_{n} q_{n}^{a} R_{n} T^{a} \bar{R}_{n}\right) \eta \\
\delta_{\eta} Q_{m} & =-\sqrt{2} i \eta u \psi_{m} \\
\delta_{\eta} R_{n} & =-\sqrt{2} i \eta u \psi_{n} \\
\delta_{\eta} \psi_{m} & =\sum_{a}-i g q_{m}^{a} \sqrt{2} \sigma \cdot x^{a} T^{a} u \bar{\eta} Q_{m}-\sqrt{2} u \bar{\eta} \dot{Q}_{m} \\
\delta_{\eta} \psi_{n} & =\sum_{a}-i g q_{n}^{a} \sqrt{2} \sigma \cdot x^{a} T^{a T} u \bar{\eta} R_{n}-\sqrt{2} u \bar{\eta} \dot{R}_{n}
\end{aligned}
$$

where one needs to use the equations of motion to cancel terms, and $\eta$ is a two-component complex constant fermion. We have written these Lagrangians in detail for future reference. Our analysis from this point will concentrate on the $n=3$ case with some relevant comments about the $n=2$ case. 


\subsection{Reduction to a Conformal Quantum Mechanics}

We will generalize the methods used by [35] for reducing a matrix model to a multidimensional Calogero type model [36]. We were inspired in our research by the proposal of [37] that the one-dimensional Calogero model described the near horizon ReissnerNordstrom extremal black holes. We were unable to confirm their proposal but found evidence from the multistrings leading to a generalized Calogero model. Rewriting the bosonic part of the $n=3$ Lagrangian with some Lagrange multipliers gives the following result,

$$
\begin{aligned}
\mathcal{L}_{n=3}^{\Lambda} & =\frac{1}{2} \sum_{a} \dot{x}_{i}^{a} \dot{x}_{i}^{a}+\sum_{m} \dot{\bar{Q}}_{m} \dot{Q}_{m}+\sum_{n} \dot{\bar{R}}_{n} \dot{R}_{n}+\operatorname{tr}\left(i \Lambda_{i j}^{(1)}\left[x_{i}, x_{j}\right]-\frac{1}{4 g^{2}} \Lambda_{i j}^{(1)^{2}}\right) \\
& -\sum_{m, a} q_{m}^{a}{ }^{2}\left(\bar{Q}_{m} x^{a} T^{a} \cdot \Lambda_{m}^{(2)}+\Lambda_{m}^{(2)}{ }^{\dagger} \cdot x^{a} T^{a} Q_{m}\right)-\sum_{n, a} q_{n}^{a}{ }^{2}\left(R_{n} x^{a} T^{a} \cdot \Lambda_{n}^{(2)^{\dagger}}+\Lambda_{n}^{(2)} \cdot x^{a} T^{a} \bar{R}_{n}\right) \\
& +\frac{1}{g^{2}}\left(\sum_{m}\left|\Lambda_{m}^{(2)}\right|^{2}+\sum_{n}\left|\Lambda_{n}^{(2)}\right|^{2}\right) \\
& -\sum_{m, a} q_{m}^{a} \bar{Q}_{m} \Lambda^{(3)^{a}} T^{a} Q_{m}-\sum_{n, a} q_{n}^{a} R_{n} \Lambda^{(3)^{a}} T^{a} \bar{R}_{n}+\frac{1}{2 g^{2}} \sum_{a}\left(\Lambda^{(3)^{a}}\right)^{2}
\end{aligned}
$$

where again most of the indices are suppressed. Integrating out the $\Lambda$ 's gives the bosonic part of the previous Lagrangian (5.3).

We derive the following equations of motion.

$$
\begin{gathered}
\ddot{x}_{i}^{a}+i\left[\Lambda_{i j}^{(1)}, x_{j}\right]^{a}+q_{m}^{a}{ }^{2} \bar{Q}_{m} T^{a} \Lambda_{m i}^{(2)}+q_{m}^{a}{ }^{2} \Lambda_{m i}^{(2)^{\dagger}} T^{a} Q_{m}+q_{n}^{a 2} R_{n} T^{a} \Lambda_{n i}^{(2)^{\dagger}}+q_{n}^{a 2} \Lambda_{n i}^{(2)} T^{a} \bar{R}_{n}=0 \\
\ddot{Q}_{m}+\sum_{a} q_{m}^{a}{ }^{2} x^{a} T^{a} \cdot \Lambda_{m}^{(2)}+\sum_{a} q_{m}^{a} \Lambda^{(3)}{ }^{a} T^{a} Q_{m}=0 \\
\ddot{R}_{n}+\sum_{a} q_{n}^{a 2} \Lambda_{n}^{(2)} \cdot x+\sum_{a} q_{n}^{a} R_{n} \Lambda^{(3)}{ }^{a} T^{a}=0
\end{gathered}
$$

Note that $g^{2}$ has the dimension of $(\text { mass })^{3}$. The low energy limit corresponds to ignoring the terms of $\mathcal{L}_{n=3}^{\Lambda}$ with coupling $\frac{1}{g^{2}}$. Doing this, we are left with some constraints.

$$
\begin{gathered}
{\left[x_{i}, x_{j}\right]=0} \\
\sum_{a} q_{m}^{a}{ }^{2} x_{i}^{a} T^{a} Q_{m}=0 \\
\sum_{a} q_{n}^{a 2} R_{n} x_{i}^{a} T^{a}=0 \\
\sum_{m} q_{m}^{a} \bar{Q}_{m} T^{a} Q_{m}+\sum_{n} q_{n}^{a} R_{n} T^{a} \bar{R}_{n}=0
\end{gathered}
$$

The "Coulomb" branch corresponds to setting all the $Q_{m}$ and $R_{n}$ to zero, whereas the "Higgs" branch corresponds to setting $x_{i}$ and the $D$ constraints (5.12) to zero. 


\subsection{The "Coulomb" Branch}

The bosonic Lagrangian on the "Coulomb" branch is

$$
\mathcal{L}_{\text {Coul }}=\operatorname{tr}\left\{\dot{x}_{i}^{2}+i \Lambda_{i j}^{(1)}\left[x_{i}, x_{j}\right]\right\} .
$$

We follow [35] in deriving a three-dimensional "spin-Calogero" [38] model. Due to the global $U\left(N_{1}\right)$ symmetry, there is a conserved matrix,

$$
V=i \sum_{i}\left[x_{i}, \dot{x}_{i}\right]
$$

Using the constraint (5.10) to diagonalize the $x_{i}$ by a time dependent unitary matrix $U$, one obtains

$$
\mathcal{L}_{\text {Coul }}=\frac{1}{2} \sum_{\alpha=1}^{N_{1}} \dot{\vec{q}}^{2}+\frac{1}{2} \sum_{\alpha \neq \beta} \frac{\tilde{V}_{\alpha \beta} \tilde{V}_{\beta \alpha}}{\left|\vec{q}_{\alpha}-\vec{q}_{\beta}\right|^{2}}
$$

where $\tilde{V}=U V U^{-1}$ and the $\vec{q}_{\alpha}$ are eigenvalues of $\vec{x}$.

One also has the relation

$$
\tilde{V}_{\alpha \beta}=i\left(\vec{q}_{\alpha}-\vec{q}_{\beta}\right)^{2} A_{\alpha \beta}
$$

where $A=\dot{U} U^{-1}$. This model becomes supersymmetric with the additional term

$$
\mathcal{L}_{\text {Coul }}^{F}=2 \operatorname{tr}\left(\tilde{\bar{\lambda}} D_{t} \tilde{\lambda}\right)
$$

where $\tilde{\lambda}=U \lambda_{L} U^{-1}$ and $D_{t}=\partial_{t}-[A$,$] . The supersymmetries which leave the action$ invariant are

$$
\begin{aligned}
\delta_{\eta} q_{\alpha}^{i} & =i \tilde{\bar{\lambda}}_{\alpha \alpha} \sigma_{i} \eta-i \bar{\eta} \sigma_{i} \tilde{\lambda}_{\alpha \alpha} \\
\delta_{\eta} \tilde{\lambda} & =\left[\left(\delta_{\eta} U\right) U^{-1}, \tilde{\lambda}\right]
\end{aligned}
$$

where one needs the equations of motion to cancel terms, and the specific form of $\delta_{\eta} U$ is not required.

The model is invariant under the conformal symmetry $S L(2, \mathbf{R})$ with action

$$
\begin{aligned}
& t^{\prime}=\frac{\mathbf{a} t+\mathbf{b}}{\mathbf{c} t+\mathbf{d}} \\
& q_{\alpha}^{i^{\prime}}\left(t^{\prime}\right)=q_{\alpha}^{i}(t)(\mathbf{c} t+\mathbf{d})^{-1} . \\
& \mathbf{a d}-\mathbf{b} \mathbf{c}=1
\end{aligned}
$$


There is also an $S O(3)$ symmetry (or $S U(2)$ including fermions). The conserved angular momentum is $J_{i j}=\sum_{\alpha}\left(q_{\alpha}^{i} \dot{q}_{\alpha}^{j}-q_{\alpha}^{j} \dot{q}_{\alpha}^{i}\right)$. The bosonic symmetry of $A d S_{2} \times S^{2}$ is $S L(2, \mathbf{R}) \times$ $S O(3)$. Since $g \sim\left(\alpha^{\prime}\right)^{-3 / 4}$ where the string tension is $\left(2 \pi \alpha^{\prime}\right)^{-1}$, the near horizon $\alpha^{\prime} \rightarrow 0$ limit in the supergravity corresponds to the $g \rightarrow \infty$ limit that we have taken to derive this theory. There is an added result that we can remove one particle far from the others $\left(\left|\vec{q}_{1}\right| \gg\left|\vec{q}_{\alpha}\right|, \alpha>1\right)$ and obtain a one particle Calogero model,

$$
\mathcal{L}_{1}=\frac{1}{2}\left|\dot{\vec{q}}_{1}\right|^{2}+\frac{L^{2}+\sum_{\beta \neq 1} \tilde{V}_{1 \beta} \tilde{V}_{\beta 1}}{2\left|\vec{q}_{1}\right|^{2}}+\mathcal{L}\left(\vec{q}_{\alpha}, \dot{\vec{q}}_{\alpha}\right)_{\alpha>1}
$$

where $L^{2}$ is the angular momentum operator for $S^{2}$. This result has previously been obtained by considering a charged particle in the supergravity background of $A d S_{2} \times S^{2}$ [39]. Note that the relativistic corrections found in [39] should result from an $\alpha^{\prime}$ expansion of the multistring theory. These corrections might give a clue to stringy excitations of the multistrings. Their results also indicate that we should have a nonlinear realization of the supersymmetry.

The bosonic Hamiltonian takes the form

$$
H_{\text {Coul }}=\frac{1}{2} \sum_{\alpha=1}^{N_{1}} \vec{p}_{\alpha}^{2}+\frac{1}{2} \sum_{\alpha \neq \beta} \frac{\tilde{V}_{\alpha \beta} \tilde{V}_{\beta \alpha}}{\left|\vec{q}_{\alpha}-\vec{q}_{\beta}\right|^{2}}
$$

One can write the generators of $S L(2, \mathbf{R})$ as

$$
\begin{aligned}
H & =H_{\text {Coul }} \\
D & =\frac{-1}{2} \sum_{\alpha} \vec{p}_{\alpha} \cdot \vec{q}_{\alpha} \\
K & =\frac{1}{2} \sum_{\alpha} \vec{q}_{\alpha}^{2}
\end{aligned}
$$

satisfying the classical Poisson bracket relations

$$
\begin{aligned}
& \{H, D\}_{P B}=H \\
& \{K, D\}_{P B}=-K . \\
& \{H, K\}_{P B}=2 D
\end{aligned}
$$

Classically, we also have the following Poisson bracket relations for $\tilde{V}$,

$$
\left\{\tilde{V}_{\alpha \beta}, \tilde{V}_{\gamma \delta}\right\}_{P B}=\frac{-i}{2}\left(\delta_{\alpha \delta} \tilde{V}_{\gamma \beta}-\delta_{\beta \gamma} \tilde{V}_{\alpha \delta}\right)
$$


Let $\tilde{V}=-\sum_{a=1}^{N_{1}^{2}-1} \tilde{V}^{a} T^{a}$. Then

$$
\left\{\tilde{V}^{a}, \tilde{V}^{b}\right\}_{P B}=f^{a b c} \tilde{V}^{c}
$$

Let us try to supersymmetrize this Hamiltonian. There is a problem here in that the variation of the constraint under the original supersymmetries of the "Coulomb" branch is nonzero when the constraint is applied.

$$
\sigma_{i} \sigma_{j} \delta\left[x_{i}, x_{j}\right] \sim[\lambda, \sigma \cdot x]
$$

Supersymmetry requires that

$$
\sigma \cdot\left(q_{\alpha}-q_{\beta}\right) \tilde{\lambda}_{\alpha \beta}=0
$$

or that $\tilde{\lambda}_{\alpha \beta}=0$ for $\alpha \neq \beta$. Examining the original Lagrangian (5.3) with $Q_{m}=R_{n}=0$, we see that we can satisfy the constraint (5.10) with nonzero fermions in the conformal limit by imposing another constraint on the fermions

$$
\left[\tilde{\bar{\lambda}}^{s}, \tilde{\lambda}^{t}\right]_{\alpha \alpha}=0
$$

all $\alpha$ where $s, t$ are $S U(2)$ spinor indices. The Gauss' law constraint (5.4) implies $\tilde{V}=$ $-[\tilde{\bar{\lambda}}, \tilde{\lambda}]$. The internal spin symmetry will be determined by the fermions, and one will obtain different models depending on the representation. Supersymmetry thus implies that the interaction $\tilde{V}=0$. We have another option. By taking a linear combination of the supersymmetries that breaks the $S U(2)$ symmetry, we can obtain a zero eigenvalue of the analog of (5.27) and partially preserve the supersymmetry with nonzero interaction. This is not an option if we are to describe a conformal dual theory to quantum gravity on $A d S_{2} \times S^{2}$. There may be a way to realize a nontrivial supersymmetry nonlinearly which is not clear at the moment.

One can rewrite the $\tilde{V}$ 's as $S U(q)$ quantum spin degrees of freedom [40]. One sets $\tilde{V}^{a}=\sum_{m=1}^{q} \psi_{m \alpha}^{\dagger} T_{\alpha \beta}^{a} \psi_{m \beta}$ where $\left\{\psi_{m \alpha}, \psi_{n \beta}^{\dagger}\right\}=\delta_{m n} \delta_{\alpha \beta}$. By defining

$$
S_{m n}^{\alpha}=\psi_{m \alpha}^{\dagger} \psi_{n \alpha}-\frac{1}{q}\left(\sum_{s=1}^{q} \psi_{s \alpha}^{\dagger} \psi_{s \alpha}\right) \delta_{m n}
$$

and using the constraint $\tilde{V}_{\alpha \alpha}=0$ to set $\sum_{s=1}^{q} \psi_{s \alpha}^{\dagger} \psi_{s \alpha}=l$ with $l$ an integer, the Hamiltonian becomes

$$
H_{\text {Coul }}=\frac{1}{2} \sum_{\alpha=1}^{N_{1}} \vec{p}_{\alpha}^{2}-\frac{1}{2} \sum_{\alpha \neq \beta} \frac{2 \operatorname{tr}\left(S^{\alpha} S^{\beta}\right)+l(l-q) / q}{4\left|\vec{q}_{\alpha}-\vec{q}_{\beta}\right|^{2}} .
$$

The spins are in the $l$-fold antisymmetric representation of $S U(q)$. One can also obtain an antiferromagnetic interaction by using bosonic oscillators.

The $n=2$ case differs from the $n=3$ case by extra global $U(1)^{2}$ symmetry. This symmetry originates from the extra BPS deformation directions for the $n=2$ multistrings. 


\subsection{The "Higgs" Branch}

The bosonic Lagrangian on the "Higgs" branch is

$$
\mathcal{L}_{\text {Higgs }}=\dot{Q}_{m} \dot{Q}_{m}+\dot{\vec{R}}_{n} \dot{R}_{n}-\sum_{m, a} q_{m}^{a} \bar{Q}_{m} \Lambda^{(3)^{a}} T^{a} Q_{m}-\sum_{n, a} q_{n}^{a} R_{n} \Lambda^{(3)^{a}} T^{a} \bar{R}_{n}
$$

where the $D$ constraints are enforced by $\Lambda^{(3)}$. The Gauss' law constraints are

$$
i \frac{d}{d t}\left(\sum_{m, a} q_{m}^{a} \bar{Q}_{m} T^{a} Q_{m}+\sum_{n, a} q_{n}^{a} R_{n} T^{a} \bar{R}_{n}\right)=\left(\sum_{m, a} q_{m}^{a} \bar{\psi}_{m} T^{a} \psi_{m}+\sum_{n, a} q_{n}^{a} \bar{\psi}_{n} T^{a T} \psi_{n}\right)
$$

If $\Lambda^{(3)}$ were time independent, this system would be a simple harmonic oscillator. One can see that $\Lambda^{(3)}$ has the dimension of $(\text { mass })^{2}$. For this action to be conformally invariant, we require that $\Lambda^{(3)}$ transforms as

$$
\Lambda^{(3)^{\prime}}=(\gamma t+\delta)^{4} \Lambda^{(3)} .
$$

If we redefine the chiral multiplets by a time dependent unitary matrix that diagonalizes $\Lambda^{(3)}$ and introduces a covariant time derivative, we have an interpretation of the square roots of the $N_{1}$ eigenvalues as time dependent inverse scale sizes of the $N_{1}$ D-particles in the transverse dimensions. To supersymmetrize the "Higgs" branch, one can perform a change of coordinates that is similar to (4.35) (assuming $8 N_{1} N_{2} N_{3} N_{4}>N_{1}^{2}$ ) so that the complex dimension is $8 N_{1} N_{2} N_{3} N_{4}-N_{1}^{2}$. The remaining massless modes have superpartners with the Lagrangian

$$
\mathcal{L}_{\text {Higgs }}^{F}=i \sum_{m} \bar{\psi}_{m} \dot{\psi_{m}}+i \sum_{n} \bar{\psi}_{n} \dot{\psi_{n}}
$$

The action is invariant under the supersymmetries

$$
\begin{aligned}
\delta_{\eta} Q_{m} & =-\sqrt{2} i \eta u \psi_{m} \\
\delta_{\eta} R_{n} & =-\sqrt{2} i \eta u \psi_{n} \\
\delta_{\eta} \psi_{m} & =-\sqrt{2} u \bar{\eta} \dot{Q}_{m} \\
\delta_{\eta} \psi_{n} & =-\sqrt{2} u \bar{\eta} \dot{R}_{n}
\end{aligned}
$$

In the limit in which the entropy estimate of section 4.3 is valid, $N_{1} \gg N_{2} N_{3} N_{4}$ so the "Higgs" branch is massive. In the $g \rightarrow \infty$ limit the "Coulomb" and "Higgs" branches appear to be decoupled from each other. At higher energies the two branches are coupled through the harmonic oscillator modes that have been ignored in the conformal limit. 


\section{Discussion}

We have conjectured that D-particles at D-brane intersections form multistrings and that these multistrings are the relevant degrees of freedom of black holes formed from these intersections. An index calculation shows that the counting of states is correct for one Dparticle interacting with one intersection. With several assumptions, one sees that these multistrings can account for the ground state entropy of the black hole. We have derived from the multistring theory a conformal quantum mechanics, the "Coulomb" branch, that exhibits some of the expected properties of supergravity on $A d S_{2} \times S^{2}$. We have also derived another conformal quantum mechanics, the "Higgs" branch, that describes part of the moduli space of D-particle-D-intersections. These two theories are coupled at higher energies. We expect also at higher energies stringy corrections to the low energy multistring theory will play a role though we currently don't know how to describe these excitations. A future goal is to determine the full effective theory of the multistrings.

It would be interesting to see whether one could reproduce the BPS spectrum of supergravity on $A d S_{2} \times S^{2}$ [1] from the conformal mechanics. 2 . The bosonic symmetries are the same, and it remains to see how and whether supersymmetry can be realized. It would also be interesting to compare correlation functions in the two theories. An even more interesting and current project is to see whether the full quantum mechanics describes the dynamics of the nonextremal Reissner-Nordstrom black hole at low energies where stringy corrections can be neglected.

\section{Acknowledgments}

I would like to thank P. Aschieri, K. Bardakci, C. Csaki, R. Dawid, S. Giddings, S. Gopalakrishna, M. Halpern, K. Hori, S. Kachru, C. Kolda, A. Konechny, B. Morariu, H. Ooguri, J. Terning, and B. Zumino for sharing some of their time to listen to and comment helpfully on some of my ideas. I am also grateful to J. Anderson, A. Friedland, S. Gopalakrishna, I. Hinchliffe, and C. Kolda for important help with various computer programs. This research was supported in part by the Director, Office of Science, Office of Basic Energy Services, of the U.S. Department of Energy under contract DE-AC03$76 \mathrm{SF} 00098$.

2 There is also related work 42 on the construction of a superstring action on $A d S_{2} \times S^{2}$. 


\section{References}

[1] G. Gibbons and P. Townsend, Phys. Rev. Lett. 71 (1993) 3754.

[2] J. Polchinski, Phys. Rev. Lett. 75 (1995) 4724.

[3] E. Witten, Nucl. Phys. B460 (1996) 335.

[4] A. Strominger and C. Vafa, Phys. Lett. B379 (1996) 99.

[5] I. Klebanov, Nucl. Phys. B496 (1997) 231.

[6] J. Maldacena, Adv. Theor. Math. Phys. 2 (1998) 231.

[7] S. Gubser, I. Klebanov, and A. Polyakov; Phys. Lett. B428 (1998) 105.

[8] E. Witten, Adv. Theor. Math. Phys. 2 (1998) 253.

[9] I. Klebanov and A. Tseytlin, Nucl. Phys. B475 (1996) 179.

[10] V. Balasubramanian and F. Larsen, Nucl. Phys. B478 (1996) 199.

[11] J. Maldacena, A. Strominger, and E. Witten; JHEP 9712 (1997) 002.

[12] J. Blum, unpublished.

[13] E. Witten, Nucl. Phys. B202 (1982) 253.

[14] J. Cardy, Nucl. Phys. B270 (1986) 186.

[15] C. Callan and J. Maldacena, Nucl. Phys. B472 (1996) 591.

[16] J. Maldacena and L. Susskind, Nucl. Phys. B475 (1996) 679.

[17] W. Taylor, Nucl. Phys. B508 (1997) 122.

[18] J. Blum, Nucl. Phys. B486 (1997) 34.

[19] J. Schwarz, Nucl. Phys. Proc. Suppl. 55B (1997) 1.

[20] A. Sen, JHEP 9803 (1998) 005.

[21] M. Gaberdiel and B. Zwiebach, Nucl. Phys. B518 (1998) 151.

[22] O. Bergman, Nucl. Phys. B525 (1998) 104.

[23] A. Sen, JHEP 9806 (1998) 007.

[24] B. Simon, Ann. Phys. 146 (1983) 209.

[25] M. Claudson and M. Halpern, Nucl. Phys. B250 (1985) 689.

[26] U. Danielsson, G. Ferretti, and B. Sundborg; Int. J. Mod. Phys. A11 (1996) 5463.

[27] S. Sethi and M. Stern, Phys. Lett. B398 (1997) 47.

[28] P. Yi, Nucl. Phys. B505 (1997) 307.

[29] S. Sethi and M. Stern, Commun. Math. Phys. 194 (1998) 675.

[30] M. Halpern and C. Schwartz, Int. J. Mod. Phys. A13 (1998) 4367.

[31] M. Porrati and A. Rozenberg, Nucl. Phys. B515 (1998) 184.

[32] G. Moore, N. Nekrasov, and S. Shatashvili; hep-th/9803265.

[33] A. Konechny, JHEP 9810 (1998) 018.

[34] J. Wess and J. Bagger, Supersymmetry and Supergravity (Princeton University Press, 1992), chapter VII, and citations there.

[35] A. Polychronakos, Phys. Lett. B408 (1997) 117.

[36] F. Calogero, J. Math. Phys. 10 (1969) 2191 and 2197; 12 (1971) 419. 
[37] G. Gibbons and P. Townsend, Phys. Lett. B454 (1999) 187.

[38] J. Gibbons and T. Hermsen, Physica D11 (1984) 337;

S. Wojciechowski, Phys. Lett. A111 (1985) 101.

[39] P. Claus, M. Derix, R. Kallosh, J. Kumar, P. Townsend, and A. Van Proeyen; Phys. Rev. Lett. 81 (1998) 455.

[40] J. Minahan and A. Polychronakos, Phys. Lett. B326 (1994) 288.

[41] J. Michelson and M. Spradlin, hep-th/9906056;

S. Corley, hep-th/9906102;

J. Lee and S. Lee, hep-th/9906105.

[42] J.-G. Zhou, hep-th/9906013. 